\title{
Missing Depth Cues in Virtual Reality Decrease Performance of Three-Dimensional Reaching Movements
}

\section{Conference Paper}

Author(s):

Gerig, Nicolas (1); Mayo, Johnathan; Baur, Kilian (1); Wittmann, Frieder; Riener, Robert; Wolf, Peter (1)

Publication date:

2017

Permanent link:

https://doi.org/10.3929/ethz-b-000218333

Rights / license:

In Copyright - Non-Commercial Use Permitted

Originally published in:

Advances in Intelligent Systems and Computing 663, https://doi.org/10.1007/978-3-319-67846-7_12 


\title{
Missing Depth Cues in Virtual Reality Decrease Performance of Three-Dimensional Reaching Movements
}

\author{
Nicolas Gerig ${ }^{1,2}$, Johnathan Mayo ${ }^{1}$, Kilian Baur ${ }^{1,2}$, Frieder Wittmann ${ }^{3}$, Robert Rie- \\ ner $^{1,2}$, and Peter Wolf ${ }^{1,2}$ \\ ${ }^{1}$ Sensory-Motor Systems Lab, Swiss Federal Institute of Technology, Zurich, Switzerland \\ ${ }^{2}$ Spinal Cord Injury Center, University Hospital Balgrist, University of Zurich, Switzerland \\ ${ }^{3}$ Rehabilitation Engineering Lab, Swiss Federal Institute of Technology, Zurich, Switzerland \\ gerig.nicolas@hest.ethz.ch
}

\begin{abstract}
Goal-directed reaching movements in three-dimensions are important for our interaction with the environment. Instrumented setups displaying virtual targets for rehabilitation training of reaching movements often provide limited depth cues, which may affect movements. This work aims to quantify effects of limited depth cues on reaching movements. We developed a virtual environment for assessing three-dimensional reaching movements that allows different depth cues to be enabled or disabled. By imposing a fixed spatial tolerance around targets for speed-accuracy trade-off, completion time normalized with the straightline distance to the target was used to measure reaching performance. In the present study, 8 (control) subjects using a typical monitor setup applied in rehabilitation were compared to 7 subjects using a head-mounted display and receiving additional depth cues, namely, hard-referenced objects of known size, motion parallax due to tracked head-movements, and stereopsis. Control subjects required on average 9.88 seconds per meter straight-line distance, while subjects using the head-mounted display required only 3.15 seconds per meter straightline distance. Additionally, movement trajectories of control subjects showed a different pattern, indicating a lack of reliable depth information. Thus, state-ofthe-art rehabilitation setups are challenging already for healthy subjects. This challenge can be reduced by improving the provided visual depth cues.
\end{abstract}

Keywords: Head-Mounted Display, Perception, Reaching Assessments, UpperArm Rehabilitation

\section{Introduction}

Patients suffering from spinal cord injury or stroke often have a reduced muscle function or muscular control, impairing the ability to perform goal-directed reaching movements [3]. Goal directed reaching is an essential movement component to enable grasping, moving, or manipulating objects [2]. Therefore, reaching movements are of great interest during rehabilitation for restoring natural function, learning compensatory strategies, or to quantify impairments [3]. 
Augmenting reaching movements with instrumented setups and virtual reality is beneficial, since the labor-intensive work of placing or indicating targets can be moved away from a human therapist into virtual reality. Additionally, virtual reality can increase motivation [6], which may well be necessary due to the repetitive and boring nature of such reaching movement trainings.

To plan and perform natural reaching movements, human subjects must have clearly perceived the target's position in three-dimensional space. For perceiving depth, i.e. the distance from an object to the human, humans rely on a variety of different depth cues [8]. However, when indicating reaching movements within virtual reality, some of these depth cues are typically not available. For example, oculomotor cues that provide depth information due to eye muscular activity are not used, since the distance to the focused virtual reality display is not the distance to the object. Recent studies in instrumented arm rehabilitation with three-dimensional movements involve setups using typical twodimensional computer monitors (e.g. see [4, 5, 11]). These setups also do not provide stereopsis by separate images for each eye, or motion parallax by rendering dependent on the viewer's perspective. Other depth cues, such as linear perspective, occlusion, and texture gradients are easy to recreate with computer graphics, and were typically provided. The relative importance of different depth cues has been shown to depend on the task [1], but for rehabilitation-typical reaching movements we have not found investigations of the effects of the different depth cues. Knowledge on the effect of such missing depth cues is crucial for designing virtual reality environments for reaching therapy, and for understanding impairment quantifications obtained with such virtual reality environments.

Recent development and releases of commercially available consumer grade virtual reality systems with head-mounted displays (HMDs, e.g. HTC $^{1}$ Vive, Oculus ${ }^{2}$ Rift, Sony ${ }^{3}$ PlayStation VR) provide affordable solutions to provide both stereopsis and motion parallax. Besides the provided depth cues by HMDs, existing setups do typically not fully exploit the retinal image size depth cue. Often, only an approximate object size is known contextually by the subject (e.g. approximate size of a fruit to be grasped), but no hard-reference of exact object size is provided at both hand avatar and reaching target. Therefore, this work aims to quantify effects of typically missing depth cues on three-dimensional reaching movements. In other words, we want to demonstrate the open potential between current state-of-the-art rehabilitation setups and what we can technically realize with a HMD for performing three-dimensional reaching movements.

High Tech Computer Corporation (HTC), Taoyuan, Taiwan

Oculus VR LLC, Menlo Park, California, USA

Sony Corporation, Minato, Tokyo, Japan 


\section{Methods}

\subsection{Apparatus}

The experiment setup consisted of a desktop computer (Windows $10^{4}$, i5-6600K $\mathrm{K}^{5} \mathrm{CPU}$ @ $3.5 \mathrm{GHz}, \mathrm{GTX}^{6} 1080 \mathrm{GPU}$ ), a desktop monitor for the control group (HP LP2065, 20", 1600x1200@85 Hz), and the HTC $\mathrm{HT}^{1}$ Vive virtual reality system (see Fig. 1). The HTC $^{1}$ Vive consists of a head-mounted display $\left(1080 \times 1200\right.$ per eye @ $90 \mathrm{~Hz}, 110^{\circ}$ horizontal field of view, $0.6 \mathrm{~kg})$, a handheld controller $(0.2 \mathrm{~kg})$, and the optical tracking system Lighthouse ${ }^{7}$ offering "sub-millimeter accuracy" and between " $250 \mathrm{~Hz}$ to $1 \mathrm{kHz}$ " update rates [10]. Virtual reality and data logging (at approximately $1 \mathrm{kHz}$ ) were realized in a custom-made Unity ${ }^{8}$ game using the OpenVR [9] library.

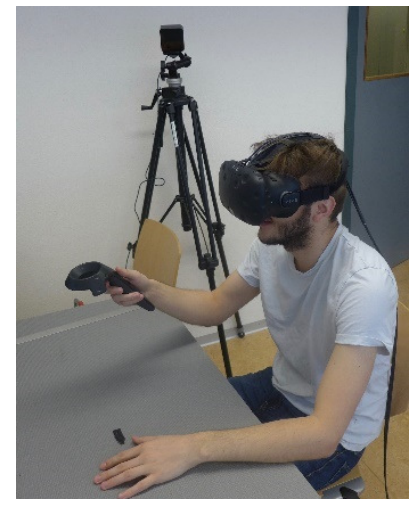

Fig. 1. User wearing the HTC Vive head-mounted display, holding a handheld controller, and a

"Lighthouse" tracking device on a tripod in the back.

\subsection{Task and Protocol}

The subjects had to move a cylindrical pointer $(0.05 \mathrm{~m}$ length, $0.01 \mathrm{~m}$ diameter) from a resting position on the table towards spherical targets and keep a part of the pointer inside the sphere for a two second dwell time (see Fig. 2). The target sphere was displayed as a semitransparent yellow table tennis ball $(0.04 \mathrm{~m}$ diameter). If any part of the pointer was within the target sphere, the pointer color changed from pink to green.

\footnotetext{
4 Microsoft Corporation, Redmond, Washington State, USA

5 Intel Corporation, Santa Clara, California, USA

6 Nvidia Corporation, Santa Clara, California, USA

7 Valve Corporation, Bellevue, Washington State, USA

8 Unity Technologies, San Francisco, California, USA
} 


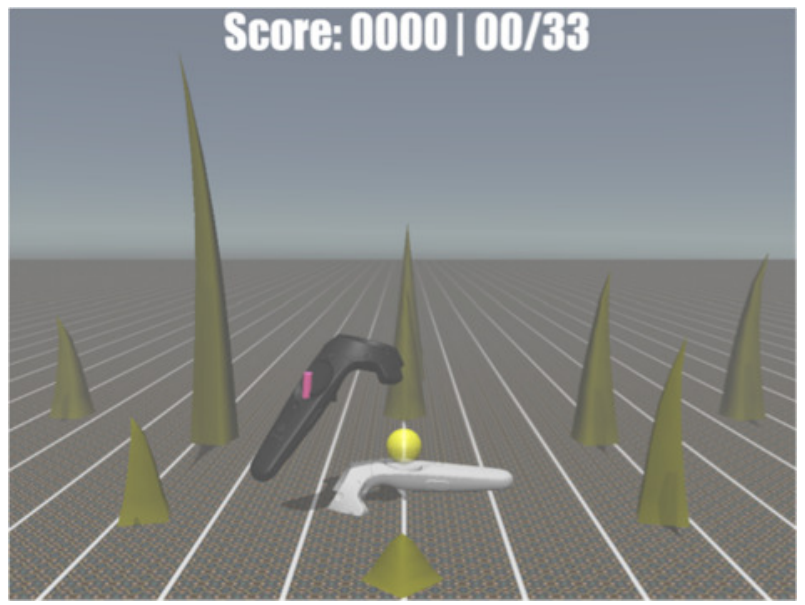

Fig. 2. Virtual reality rendering of the task with all realized monocular depth cues, the cylindrical pointer in pink, and the starting position indicated by the yellow target sphere.

The quantitative outcome of the individual reaching movements was time to completion. Subjects were instructed to perform the task as fast as possible. The controlled spatial tolerance given by the $0.04 \mathrm{~m}$ sphere was considered to control speed-accuracy trade-off and justify time to completion as a reasonable performance metric.

In total, 33 targets were presented to each subject. Targets were distributed throughout a quarter-sphere of $0.32 \mathrm{~m}$ radius. The first 4 targets were placed at the extremes of the workspace and served to test the participants' range of motion and familiarize them with the task procedure. All subjects received the same targets in the same order.

At the start of the trial, the target sphere was displayed at the starting position. Once the subject reached the starting position, a $15 \mathrm{~s}$ countdown was started. The first $10 \mathrm{~s}$ of this countdown were provided as a resting period before presenting the next target to avoid fatigue. During this first $10 \mathrm{~s}$, the countdown from 15 to 6 was visually displayed. The next target was presented $5 \mathrm{~s}$ before the countdown ran out, to allow the subject to locate the target in space and plan the movement. To avoid occlusion of the scenery, the start countdown from 5 to 0 was not displayed visually. Instead the last $3 \mathrm{~s}$ $(3,2,1)$ were indicated with a $440 \mathrm{~Hz}$ sounds of 0.2 s duration each, and the start sound at the end of the countdown with a frequency of $880 \mathrm{~Hz}$ and a duration of $0.2 \mathrm{~s}$ duration, mimicking the start sounds commonly used for car races. The start sound was accompanied by a small vibration impulse realized with the handheld. False starts, i.e. moving out of the $0.04 \mathrm{~m}$ diameter sphere around the starting position before the start signal, were indicated by removal of the target sphere and excluded from analysis. After false start or task completion, the start position was indicated with the target sphere. Once the subject returned to the start position, the next $15 \mathrm{~s}$ countdown was started.

To keep subjects motivated, they received performance feedback about their last reaching movement during resting time. The score $S_{i}$ of movement $i$ was calculated as

$$
S_{i}=300 \cdot \frac{d_{i}}{\Delta t_{i}}
$$

where $d_{i}$ was the straight-line distance between start and target position in meter, and 
$\Delta t_{i}$ the time to task completion in seconds (without the last two seconds of dwell time). The gain was chosen, such that typically a two to three-digit integer score could be displayed.

\subsection{Virtual Environment and Depth Cues}

The virtual environment for the reaching tests was designed to provide minimal rendered objects and distraction, while allowing to toggle as many different visual depth cues as possible (see Fig. 2). Visual depth cues (see e.g. [8]) that were realized with our setup, their implementation in the virtual scenery, and the group definitions are summarized in Table 1. The control group did not receive the visually rendered handheld and ghost handheld as hard-referenced objects of known size. Their only object of known size was the table tennis target sphere, to emulate the condition that is commonly used in virtual reality enhanced rehabilitation.

Table 1. Depth cues, their implementation and group definition.

\begin{tabular}{|c|c|c|c|}
\hline Depth Cue & Implementation & $\begin{array}{l}\text { HMD } \\
\text { group }\end{array}$ & $\begin{array}{l}\text { Control } \\
\text { group }\end{array}$ \\
\hline Aerial Perspective & Virtual fog. & & \\
\hline Linear Perspective & White parallel lines on the scenery ground. & & \\
\hline $\begin{array}{l}\text { Motion Parallax } \\
\text { (Scenery Motion) }\end{array}$ & $\begin{array}{l}\text { Constant virtual wind moving blades of grass, } \\
\text { closer blades of grass appear to be moving } \\
\text { more. }\end{array}$ & & \\
\hline Occlusion & $\begin{array}{l}\text { Blades of grass, that may partially occlude each } \\
\text { other, target, or pointer. }\end{array}$ & & \\
\hline Shadows & $\begin{array}{l}\text { Shadows of grasses, pointer, target, handhelds, } \\
\text { and ghost handheld. }\end{array}$ & & \\
\hline Texture Gradients & $\begin{array}{l}\text { Grey texture at scenery ground, where a more } \\
\text { detailed rendering is visible closer to the sub- } \\
\text { ject. }\end{array}$ & & \\
\hline $\begin{array}{l}\text { Motion Parallax } \\
\text { (Subject Motion) }\end{array}$ & $\begin{array}{l}\text { Virtual scene is rendered from the current view- } \\
\text { ing position and angle, which can be tracked by } \\
\text { the HMD, close objects seem to move more. }\end{array}$ & & \\
\hline Retinal Image Size & $\begin{array}{l}\text { Object of known size at both, pointer position } \\
\text { by showing virtual black handheld, and at target } \\
\text { position by white ghost handheld. }\end{array}$ & & \\
\hline Stereopsis & $\begin{array}{l}\text { Individual screens for each eye in the HMD } \\
\text { providing different images for both eyes. }\end{array}$ & & \\
\hline
\end{tabular}




\section{$2.4 \quad$ Subjects}

In total, 17 healthy volunteers were recruited and pseudo-randomly assigned to the groups. The pseudo-randomization was performed based on a randomly permuted assignment list, ensuring approximately equal group sizes. The experimental protocol and subject recruitment was approved by the ETH Zurich Ethics Commission (EK 2014-N21).

Subjects did not have any experience performing the test task prior to the experiment. However, three subjects from the lab correctly guessed the experimental hypothesis during the trial. All subjects had a range of motion compatible with the experiment, were 18 to 40 years old, participated in sports at least half an hour per week, and reported no non-corrected sight or hearing defects.

All subjects had to perform the task with their right hand. Subject handedness was determined with the Edinburgh Handedness Inventory [7]. One left-handed and one ambidextrous participant were excluded from data analysis. From the remaining 15 subjects, 8 were assigned to the control group.

\subsection{Statistical Evaluation}

The primary outcome variable time to task completion was normalized with the straight-line distance between start and target position. For each subject, one average time over distance was calculated. The 7 data points from HMD were compared against the 8 from the control group. First, a Levene test for the assumption of equal variance was performed. In case variance between the groups was significantly different, a nonparametric Kruskal-Wallis test was performed instead of one-way ANOVA. The significance level was fixed to $\alpha=0.05$.

\section{Results}

The HMD group completed the reaching tasks on average in $\mu_{h m d}=3.15$ seconds per meter straight-line distance, the control group took in average $\mu_{c t r l}=9.88 \mathrm{~s} / \mathrm{m}$ (Fig. 3). The Levene test for the assumption of equal variance failed $\left(F_{1,13}^{\text {levene }}=15.9, p=\right.$ $0.0015)$. The Kruskal-Wallis test showed significant group differences $\left(F_{1,13}^{\text {chisq }}=10.5\right.$, $p=0.0012$ ). 


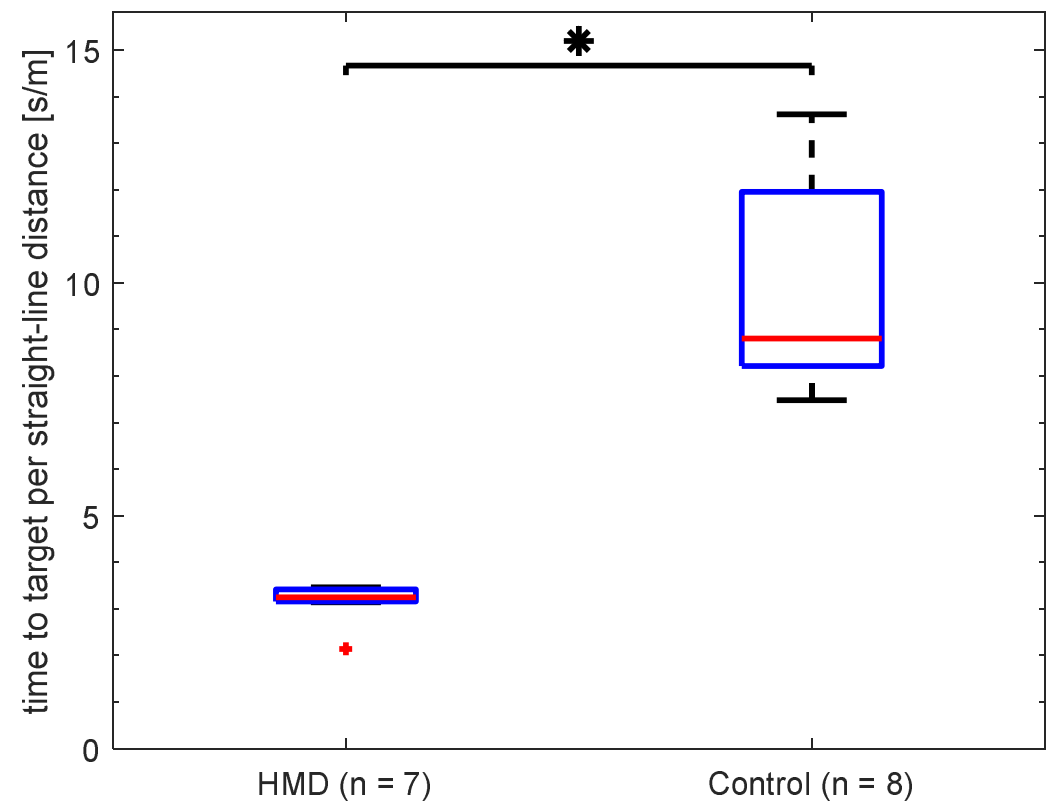

Fig. 3. Between group comparison of subject average time to target normalized by the straightline distance, with group median as horizontal red lines, 50\% coverage intervals as blue boxes, $\pm 2.7 \sigma$ or $99.3 \%$ coverage intervals with black whiskers, one outlier as a red cross, and the significant group difference with a starred black horizontal bar.

The $30^{\text {th }}$ target position served as an illustrative example of differences in movement trajectories. While subjects in the HMD group showed relatively direct handheld trajectories, the control subjects failed to do so (Fig. 4).

A total of 16 false starts occurred that were excluded from analysis. The HMD group showed 3 false starts out of 203 movements ( 7 subjects, 29 evaluated targets) and the control group showed 13 false starts out of 232 evaluated movements ( 8 subjects, 29 evaluated targets). 
target number $30: 0 / 15$ false starts.

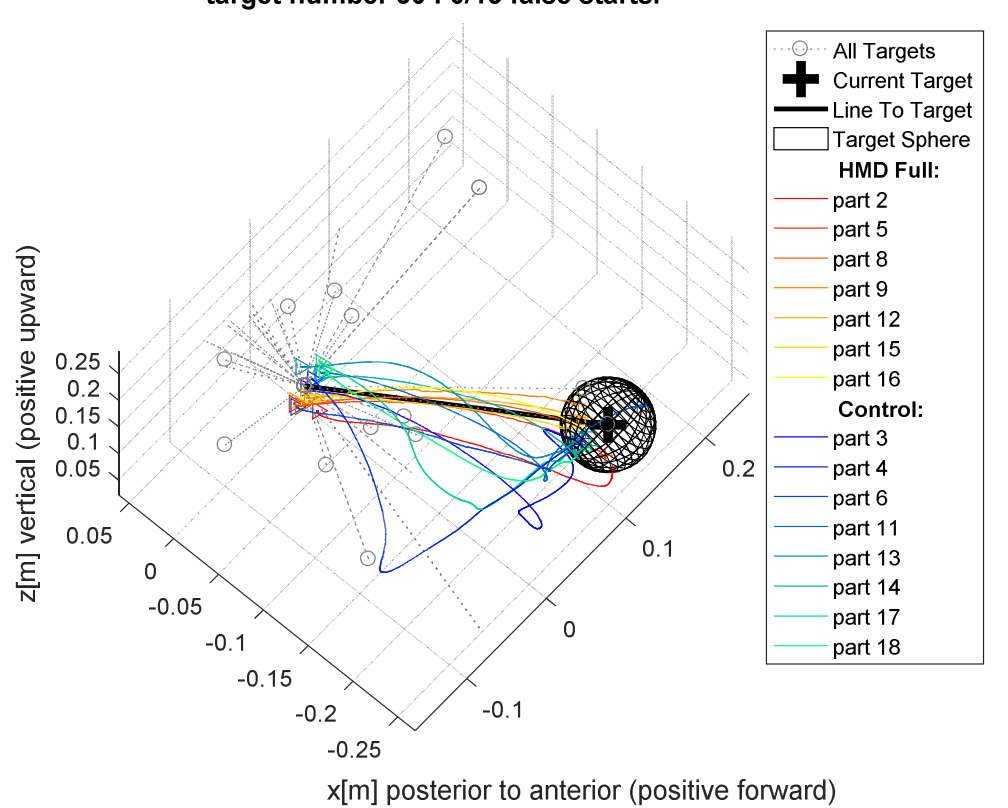

$y[m]$ lateral (positive left)

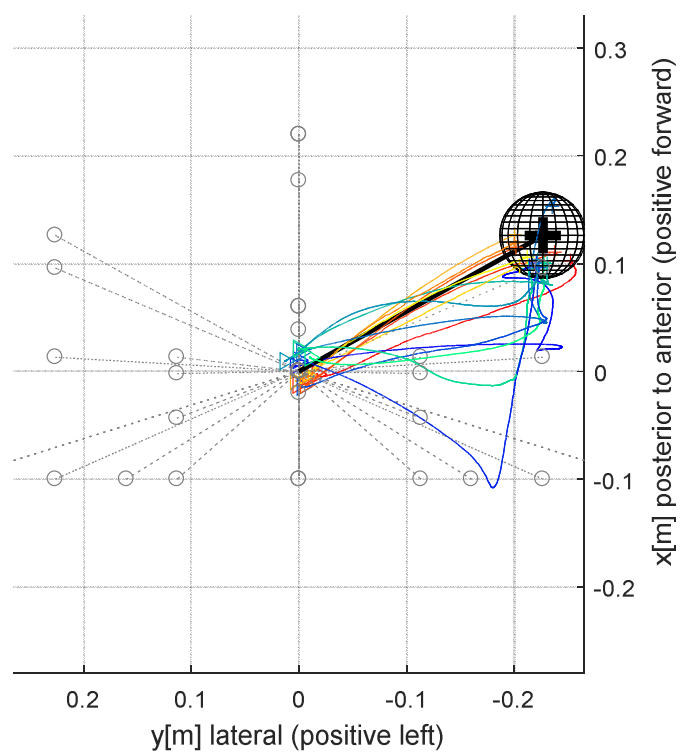

Fig. 4. Illustration of movement trajectories in 3D view (top) and top-down view (bottom) for target number 30. HMD subjects are illustrated in colors from red to yellow and control subjects from blue to green. 


\section{Discussion}

The observed differences in three-dimensional reaching movement performance (Fig. 3) between the group with the Head-Mounted Display (HMD) and the control group with the standard screen exceeded our expectations. The three-dimensional reachingtask was designed to resemble reaching movement training or impairment assessments from rehabilitation. Therefore, the task was supposed to be very simple for healthy subjects. However, we could show that already healthy subjects perform very unnatural movement trajectories (Fig. 4) in three-dimensional reaching if a rehabilitation typical two-dimensional screen setup is used. These unnatural and longer movements partly explain the observed increased time to completion, however, do not mitigate the problem itself. In rehabilitation and impairment assessments, the goal is to train or measure natural reaching, but for a typical screen setup already healthy subjects show difficulties to perform natural movement trajectories. This finding may be less critical for rehabilitation training, where high-intensity training and a high number of repetitions are of primary concern. However, this finding might be critical for impairment assessments where these additional difficulties arising from location perception could mask impairment effects.

Possible limitations that could be partially responsible for the observed performance difference are motivation bias and incomplete subject blinding. The subjects were recruited for a study testing a new virtual reality system. Control subjects were not wearing the HMD but using a two-dimensional screen, which may be frustrating when expecting to test a new virtual reality system. In contrast, using immersive virtual reality is assumed to be motivating, especially for subjects in the HMD group that had no or only little experience with head-mounted displays before. Additionally, subjects may have correctly guessed the research question, and subjects not using the HMD may have easily guessed their assignment to control. However, we consider it unlikely that the movement trajectory differences shown by the control group (Fig. 4) are purely based on these limitations.

Another potential contributor for our big group difference is that the HMD setup was very immersive and blocked the real-world view completely. Eventually, subjects in the HMD group could purely rely on subconscious motor control, meaning they only had to actively think about the goal of the movement but not about movement execution. However, subjects in the control group eventually used visuomotor control consciously to control and synchronize both their hand movements in real world and the displayed movements on the screen. Thus, this additional mental load of actively using visuomotor control and the need of learning a visuomotor mapping from real to virtual world could be partially responsible for slower movements and therefore for their higher times to complete the reaching movements.

However, the trajectories performed (Fig. 4) seem to indicate that a major reason for the observed group differences is a behavioral difference in reaching strategy. Compared to HMD subjects that move close to a straight-line, subjects of the control group seem to first move to a correct two-dimensional position and then move back- or forwards in depth until they hit the target. Since hitting the target was visualized with the pointer changing its color, this movement strategy can be considered failsafe, meaning 
even without any depth perception this strategy would allow completing the task. Subjects in the control group seemed to have limited trust or benefit of the provided depth cues and therefore fell back on this less efficient but failsafe strategy. Thus, rehabilitation typical screen setups seem to provide serious challenges for depth perception, which can heavily affect subjects' reaching movements and impede their performance.

Eventually, subjects could learn to use the provided depth cues by practice and would then move away from this failsafe strategy. This means that impairment assessments based on three-dimensional reaching movements with such a setup would then at least partially measure virtual reality specific perception instead of the ability to perform reaching movements.

The rather high variability in reaching performance of control participants was partly expected (Fig. 3). When performing challenging movement experiments with humans, between participant variability in motivation, dexterity, cognitive abilities to find successful strategies, and different previous experience levels of perceiving virtual worlds may accumulate to a high variability in the measured performance. For participants performing under less challenging conditions, these participant preconditions may lose their impact on the performance metrics. E.g. if the depth perception in the HMD condition was not requiring any conscious mental load, variability in motivation or cognitive abilities have less effect on the measured performance. Participants using the HMD showed very low between subject variability in performance and significantly less than control. This might be an indication that the HMD group shows flooring effects, where depth perception requires no conscious mental demand beyond what all subjects are all very skilled to do from real life.

Based on our observations in this study, we cannot attribute which of the additional depth cues (Table 1) was responsible for, or mainly contributed to, the observed group differences. Since we have not provided the same retinal image size cues to both groups, we do not want to conclude on the benefit of HMDs in general. Our study was designed to show the open potential for improved depth cues between current state-of-the-art setups and what we could technically realize. Since state-of-the-art rehabilitation environments do typically not contain objects with a hard-referenced known size, i.e. the handheld in our case, we have decided to avoid this cue for control. Nevertheless, our virtual environment included the other realizable depth cues and was designed with the goal to maximize clarity of each cue. Therefore, we are confident that our control group received better or at least equal depth information than subjects using typical state-ofthe-art rehabilitation setups.

\section{Conclusion}

Missing depth cues in state-of-the-art monitor setups may change strategy and heavily impede performance when performing three-dimensional reaching movements with virtual targets. Impairment assessments obtained by reaching movements using such conventional monitor setups should be interpreted with care. Thus, we conclude that 
state-of-the-art rehabilitation setups leave a great open potential to make three-dimensional reaching movements in virtual reality more natural by providing better depth cues.

If head-mounted displays are necessary, or if optimizing or adding additional depth cues to the virtual reality environments is sufficient to exploit this potential stays an open question. Ranking the different depth cues' importance for three-dimensional reaching movements is subject of further research.

\section{References}

1. Arsenault, R. and Ware, C. 2004. The importance of stereo and eye-coupled perspective for eye-hand coordination in fish tank VR. Presence: Teleoperators and Virtual Environments. 13, 5 (2004), 549-559.

2. Birkenmeier, R.L. et al. 2010. Translating animal doses of task-specific training to people with chronic stroke in 1-hour therapy sessions: a proof-of-concept study. Neurorehabilitation and neural repair. (2010).

3. Keller, U. et al. 2015. Robot-assisted arm assessments in spinal cord injured patients: a consideration of concept study. PloS one. 10, 5 (2015), e0126948.

4. Klamroth-Marganska, V. et al. 2014. Three-dimensional, task-specific robot therapy of the arm after stroke: a multicentre, parallel-group randomised trial. The Lancet Neurology. 13, 2 (2014), 159-166.

5. Meyer-Heim, A. and Hedel, H.J. van 2013. Robot-assisted and computer-enhanced therapies for children with cerebral palsy: current state and clinical implementation. Seminars in pediatric neurology (2013), 139-145.

6. Mihelj, M. et al. 2012. Virtual rehabilitation environment using principles of intrinsic motivation and game design. Presence: Teleoperators and Virtual Environments. 21, 1 (2012), 1-15.

7. Oldfield, R.C. 1971. The assessment and analysis of handedness: the Edinburgh inventory. Neuropsychologia. (1971).

8. Riener, R. and Harders, M. 2012. Virtual reality in medicine. Springer.

9. Unity Technologies OpenVR, https://docs.unity3d.com/manual/vrdevices-openvr.html, last accessed April 13th, 2017.

10. Valve Corporation SteamVR-Tracking, https://partner.steamgames.com/vrtracking/, last accessed April 13th, 2017.

11. Wittmann, F. et al. 2016. Self-directed arm therapy at home after stroke with a sensor-based virtual reality training system. Journal of NeuroEngineering and Rehabilitation. 13, 1 (2016), 75. 